\title{
Cyclone track forecasting based on satellite images using artificial neural networks
}

Rita Kovordanyi and Chandan Roy

Linköping University Post Print

Tweet

N.B.: When citing this work, cite the original article.

Original Publication:

Rita Kovordanyi and Chandan Roy, Cyclone track forecasting based on satellite images using artificial neural networks, 2009, ISPRS journal of photogrammetry and remote sensing (Print), (64), 6, 513-521.

http://dx.doi.org/10.1016/j.isprsjprs.2009.03.002

Copyright: Elsevier

http://www.elsevier.com/

Postprint available at: Linköping University Electronic Press

http://urn.kb.se/resolve?urn=urn:nbn:se:liu:diva-52892 


\section{Cyclone Track Forecasting Based on Satellite Images Using Artificial Neural Networks}

Rita Kovordányi*

*Corresponding author

Linköping University

SE-581 83 Linköping, Sweden

Tel: +4613281430

Fax: +4613142231

E-mail: ritko@ida.liu.se
Chandan Roy

Linköping University

SE-581 83 Linköping, Sweden

E-mail: charo@ida.liu.se

Many places around the world are exposed to tropical cyclones and associated storm surges. In spite of massive efforts, a great number of people die each year in cyclone attacks. To mitigate the damage caused by cyclones in the future, it is crucial that improved cyclone forecasting techniques are developed. The present research is a step towards automatic cyclone forecasting using artificial neural network techniques to interpret NOAA-AVHRR satellite images. A multilayered neural network, resembling the human visual system, was trained to forecast the movement direction of cyclones. After training, the network produced correct directional forecast for $95 \%$ of the training images. In addition, the network was able to handle $95 \%$ of novel test images, thus showing a good generalization capability. These results are promising and indicate that multi-layered neural networks could be further developed into an effective tool for cyclone track forecasting based on remote sensing data. Future work includes extension of the present network to handle a wide range of cyclones and to take into account supplementary factors, such as wind speeds around the cyclone, water temperature, air moisture, and air pressure.

Keywords: Cyclone Track Forecasting, Artificial Neural Networks, Multi-Layer Networks, Receptive Field, Remote Sensing, Geohazards

\section{Introduction}

A tropical cyclone is an area of low pressure which develops over tropical or subtropical waters. These systems form over all tropical oceans with the exception of the South Atlantic and the eastern South Pacific east of about $140^{\circ} \mathrm{W}$ longitude. There are seven tropical cyclone basins, where tropical cyclones form on a regular basis:

1. Atlantic basin

2. Northeast Pacific basin

3. North Indian basin

4. Southwest Indian basin

5. Southeast Indian/ Australian basin

6. Australian/ Southwest Pacific basin, and

7. Northwest Pacific basin

In their most intense state these storms are called hurricanes in the Atlantic, typhoons in the western North Pacific and cyclones in the Bay of Bengal. These low-pressure systems draw their energy from the very warm sea-surface waters. As the warm, moist air spirals counterclockwise 
in toward the centre, the wind speeds increase, reaching their maximum values in the region surrounding the almost calm centre of the cyclone.

As most cyclones are formed in the tropical seas and at the same time the density of population is greatest in the tropical regions, cyclones constitute a major hazard for a large number of people around the world (Cerveny and Newman, 2000). Due to this, cyclones occupy a prominent place among the world's worst meteorological disasters. Some of the more known examples include the Bangladesh cyclone of November 12, 1970 and May 24, 1985 (both crossed the coast at Chittagong), hurricane Camille that hit USA on August 17, 1969 and cyclone Tracy that swept over the Australian coast on December 25, 1974; and recently hurricane Katrina; these cyclones have each caused innumerable fatality and immense damage in property. Frequent attacks of less intensive cyclones (having less wind velocity and lower surge levels) continue to cause human casualties and considerable economic damage to tropical countries. For these reasons substantial resources have been devoted around the world to research, forecasting and socioeconomic preparedness for cyclones and cyclone generated disasters. The present paper focuses on automating the forecasting of cyclone track and thereby providing a more reliable basis for early warning systems.

\subsection{Existing techniques for cyclone forecasting}

Tropical Cyclone (TC) forecasting involves the prediction of several interrelated features, including the track of the cyclone, its intensity, resulting rainfall and storm surge and, of course, the areas threatened. Among these features, forecasting the future track and intensity of tropical cyclones are considered to be the most important because the casualties and loss of property depend critically on these two features (Table 1).

Table 1. A sample of techniques used in various offices to forecast cyclone tracks (adapted from McBride and Holland, 1987).

\begin{tabular}{|c|c|c|c|c|c|c|c|c|c|c|c|c|}
\hline \multirow[t]{2}{*}{ Office } & \multicolumn{12}{|c|}{ Techniques } \\
\hline & \multicolumn{3}{|c|}{ Subjective } & \multirow[t]{2}{*}{ Analogue } & \multirow[t]{2}{*}{ Steering } & \multicolumn{4}{|c|}{ Statistical } & \multicolumn{2}{|c|}{ Dynamical } & \multirow[t]{2}{*}{ Empirical } \\
\hline Japan & $\mathrm{X}$ & $\mathrm{X}$ & $\mathrm{X}$ & & & $\mathrm{X}$ & $\mathrm{X}$ & & & & $\mathrm{X}$ & \\
\hline Hong Kong & $\mathrm{X}$ & $\mathrm{X}$ & & & $\mathrm{X}$ & $\mathrm{X}$ & $\mathrm{X}$ & & & & & \\
\hline Philippines & $\mathrm{X}$ & $\mathrm{X}$ & & & & $\mathrm{X}$ & $\mathrm{X}$ & & & $\mathrm{X}$ & & \\
\hline Miami & $X$ & $\mathrm{X}$ & & $\mathrm{X}$ & & $X$ & $\mathrm{X}$ & & $\mathrm{X}$ & $X$ & $\mathrm{X}$ & \\
\hline India & $\mathrm{X}$ & $\mathrm{X}$ & & $X$ & & $\mathrm{X}$ & & & & & & \\
\hline Brisbane & $\mathrm{X}$ & $\mathrm{X}$ & $\mathrm{X}$ & $\mathrm{X}$ & $\mathrm{X}$ & & $\mathrm{X}$ & & & & & \\
\hline Fiji & $\mathrm{X}$ & $\mathrm{X}$ & & & & & & & & & & \\
\hline Reunion & $\mathrm{X}$ & $\mathrm{X}$ & & & & & & & & & & \\
\hline Mauritius & $X$ & $\mathrm{X}$ & & & & & & & & & & \\
\hline Mozambique & $\mathrm{X}$ & $\mathrm{X}$ & & & & & & & & & & \\
\hline Madagascar & $\mathrm{X}$ & $\mathrm{X}$ & & & & & & & & & & \\
\hline Darwin & $\mathrm{X}$ & $\mathrm{X}$ & $\mathrm{X}$ & $\mathrm{X}$ & $\mathrm{X}$ & & $\mathrm{X}$ & & & & & \\
\hline Guam & $\mathrm{X}$ & $\mathrm{X}$ & & $\mathrm{X}$ & $\mathrm{X}$ & $\mathrm{X}$ & & $\mathrm{X}$ & & & $\mathrm{X}$ & $\mathrm{X}$ \\
\hline
\end{tabular}

Subjective assessment: This technique includes synoptic reasoning, evaluation of expected changes in the large-scale surrounding flow fields and subjective evaluation of the cyclone's steering current.

Analogue forecasts: Designated features of the cyclone, such as its latitude, longitude, intensity, maturity, and past motion, are compared to those of all previous cyclones in the same region to select one or more analogues. The cyclone movement is then derived from the previous development of tracks of the analogues. 
Steering current: The cyclone's steering current is determined using analysis of winds at specified points and altitudes around the cyclone. The actual forecast can be based on simple regression analysis, or on analysis of the advection and propagation of winds, incorporating linear interactions between the vortex and the background absolute vorticity (Holland, 1984).

Statistical technique: All the statistical forecasting techniques are based on regression analysis. Here historical patterns of previous storms, such as cloud patterns, intensity development, and previous actual track are adapted and applied to the present storm.

Dynamical: These techniques are based on numerical integration of mathematical equations that approximate the physical behavior of the atmosphere. The technique looks different when it is applied on a regional or global scale.

Empirical: A skilled meteorologist has often developed an ability to detect overall patterns in climatological conditions and can assess how these may affect cyclone development. Manual forecasts made by a skilled meteorologist may therefore be a good complement to other forecasting techniques. The success of this technique critically depends on the experience of the forecaster.

The National Hurricane Center (NHC) of USA and Australia Bureau of Meteorology (2007) identify the following additional techniques for cyclone movement forecasting:

Persistence: This technique is useful for short-term forecasts, and assumes that the cyclone will maintain its recent track. This technique is often used in combination with other techniques.

Satellite-based techniques: In this technique track and intensity are forecasted based on the cloud pattern associated with the cyclone. Generally the outer cloud bands of cumulonimbus clouds indicate the future direction, and the cloud pattern surrounding the cyclone eye indicates the future intensity of the cyclone.

Hybrid: Meteorological centers round the world often employ a combination of techniques to get a more accurate track forecast, for example, combining elements of two or more of the above techniques. The elements are blended as a weighted sum, where the weights are based on past performance of each forecasting technique (Naval Research Laboratory, 1999).

\subsubsection{Automated forecasting}

Like other forecasts, tropical cyclone forecasts are not free from error. Error in the initial position and motion of the tropical cyclone can have an impact on the accuracy of subsequent forecasts. Errors can also arise from a lack of full understanding of the mechanisms behind the formation and growth of tropical cyclones and from the limitations of the forecasting techniques themselves. Mean track forecast error is typically smaller for lower latitude cyclones moving westward than for higher latitude cyclones in westerly winds and for those cyclones which are recurving. In general, mean track forecast errors tend to increase with the forecast period and can be as much as $30 \%$ of the cyclone movement within this same period. Hence, the forecasted track can deviate from the cyclone's actual track by as much as 20 degrees.

Due to the inherent complexity of the possible factors affecting cyclone development, meteorological offices around the world try to automate much of the work involved in cyclone forecasting. The Automated Tropical Cyclone Forecasting System (ATCF), developed by the Naval Research Laboratory (NRL) in Monterey, California, is an example of automated forecasting system (Naval Research Laboratory, 1999). This computer-based application is intended to automate and optimize much of the tropical cyclone forecasting process. It provides a means for tracking, and intensity forecasting, as well as constructing messages, and disseminating warnings.

\subsubsection{Satellite-based techniques}

During the 1980s, forecasting of the track and intensity of tropical cyclones was mainly based on statistical (regression) methods using general meteorological data. Later, in the early 1990s, 
remote sensing techniques were starting to be used successfully for cyclone forecasting (Marshall et al., 2002; Wells, 1987). Development of new techniques, such as the generation of high resolution atmospheric motion vectors (AMVs) from satellite images, and four dimensional variational assimilation (4D-VAR) have reduced the error in forecasting a cyclone's track and intensity (Marshall, et al., 2002).

Images of different channels obtained from weather satellites have their specialized use in track and intensity forecasting of tropical cyclones. For example, satellite images within the thermal infrared (IR) band can be used to forecast and analyze the cyclone's intensity (Kossin, 2003). On the other hand, data from Advanced Microwave Sounder Units having better horizontal resolution and vertical temperature sounding abilities provide an improved basis for temperature estimation compared to conventional Microwave Sounding Units and IR satellite images (Knaff et al., 2000), while Lau and Crane (1997) and Kishtawal et al. (2005) measured the intensity of tropical cyclones based on satellite images and data from a Thermal Microwave Imager using non-linear data fitting.

Satellite techniques can be used for forecasting both cyclone intensity and cyclone track. TC development can be analyzed by studying the cloud patterns and determining how they change with time. Repeated observations of a TC provide information on the intensity and the rate of growth or decay of the storm. This method of intensity analysis is based on the degree of spiraling in the cloud bands. The more spiral the cloud pattern is the more intense is the cyclone (Dvorak, 1975). The Dvorak model of forecasting intensity is successful in most of the cases. However, the technique is based on subjective judgment and is unreliable in the sense that various weather stations round the world can arrive at discrepant results for the same cyclone. Velden et al. (1998) used a computer based algorithm named Objective Dvorak Technique (ODT) to address this problem.

\section{A new method for automated cyclone track forecasting}

One technique that has not been used for cyclone track forecasting previously is artificial neural networks (ANN). This in spite the fact that ANN-techniques have been used in other remote sensing application areas, such as road network detection (Barsi and Heipke, 2003), cloud detection (Jang et al., 2006), cloud motion detection (Brad and Letia, 2002a, 2000b), and precipitation forecasting (Hong et al., 2004; Rivolta, et al., 2006) based on aerial photographs and satellite images. One conceivable reason why ANN-techniques have not been applied to cyclone forecasting before is that it is difficult to achieve robust network performance in this complex domain.

Previous research suggests that cloud patterns surrounding the cyclone is a good indicator of the direction of cyclone movement. There is a tendency for TCs to move towards the downstream end of convective cloud bands in the outer circulation strips around the cyclone (Lajoie, 1976). Changes in the orientation of such cloud bands indicate that a similar change in cyclone direction may occur in the next 12-24 hours. Further, TCs do not continue towards, nor curve towards cumulonimbus free sectors in the outer circulation. Fett and Brand (1975) noted that rotation of gross cloud features (such as an elliptical cloud mass or a major outer band) provide a very good indication of cyclone direction changes during the next 24 hours.

The above results indicate that a cyclone's track is reflected by the shape, and relative position of surrounding cumulonimbus clouds. What is more, these features are visible in satellite images (Fig. 1). Detecting and categorizing these features using a neural network would therefore provide valuable input to automated cyclone forecasting. In this article we demonstrate that neural networks can learn to detect the clouds that are present in the satellite image and learn to recognize how these clouds are positioned relative to the cyclone. The neural network that we present exploits the overall shape and orientation of the cyclone with surrounding clouds. The output of this neural network may be used to predict the future track of the cyclone. 


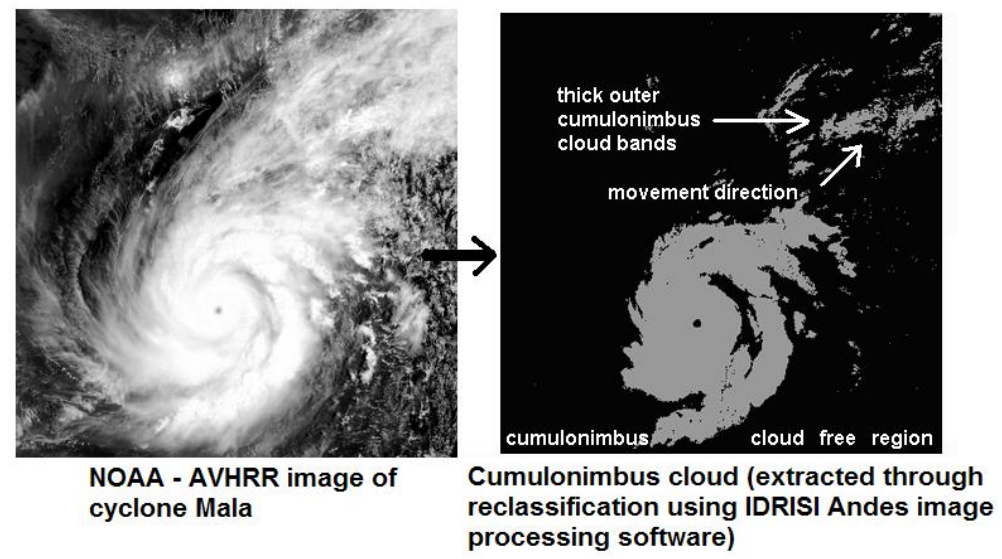

Fig. 1. Example of a satellite image showing a typical cloud pattern surrounding a cyclone. The dense cumulonimbus clouds, which are visible in the top right corner of the image, indicate a strong upstream of hot, moist air. This upstream creates a low pressure sector that fuels the cyclone and sucks it in a northeastern direction.

\subsection{Robust pattern recognition inspired by human vision}

The task for the neural network is to extract the form of dense clouds in the satellite image and recognize the overall elongated shape that is created by the cyclone in combination with the surrounding clouds. In addition, the network must produce a directional indication depending on the orientation of this overall shape.

Presently, the network is fed a low-resolution transformation of the satellite image as input (cf. Fig. 1). Additional factors that may affect the actual cyclone movement are, among other things, sea and air temperature, air pressure, and wind speeds. A practically useful indicator of future cyclone movement, used by existing forecasting systems, is the cyclone's previous movement direction. The present study is a proof of concept, meant to demonstrate the feasibility of ANNs for satellite image interpretation. Because of limited computational resources, the above additional variables were not included in the present study. This in turn entailed that the predictions made by the network reflect theoretical movement direction that can be extracted from the information contained in the satellite image.

Previous applications of neural networks in the area of remote sensing, for example, detection of the presence of clouds, and estimation of air temperatures based on satellite images deploy standard three layer feed forward networks (Brad and Letia, 2002a, 2002b; Barsi and Heipke, 2003; Hong et al., 2004; Jang et al., 2006; Rivolta et al., 2006).

Instead of the traditional three-layer feedforward network, we developed a network that is inspired by human vision. Human pattern recognition is robust in the sense that it can extract shape based on how characteristic constituent features are located relative to each other, and at the same time disregard variations in the exact location and size of the shape within the image. This is exactly what we need to do in the present study: to extract the shape of the cyclone with the surrounding cumulonimbus clouds independently of variations in the location and size of the overall cloud pattern, and to determine the orientation of this cloud pattern.

The human visual system divides visual processing into a number of transformational steps, which allows the visual system to handle the complexity of pattern recognition little by little. Transformational steps in the human visual system form a chain. In this chain, each 
transformational step communicates reciprocally with both the predecessor and successor steps (Ungerleider and Mishkin, 1982; Felleman and Van Essen, 1991). When constructing our network, we followed the example of human vision, but down-scaled the network architecture into five reciprocally connected layers, implementing four transformational steps (Fig. 2).

Previous networks in the area of remote sensing have without exception been trained using the backpropagation of error learning algorithm (Barsi and Heipke, 2003; Hong et al., 2004; Jang et al., 2006; Rivolta, et al., 2006). In contrast, humans use a combination of learning algorithms (O’Reilly, 1998). To process perceptual information, humans use model-based learning, which takes into account the statistical regularities that occur across various experiences. In addition, to be able to perform tasks intelligently, humans use regularities in their experiences as cues that help them decide how to interpret the situation and how to act, so that similar situations will tend to elicit similar reactions. To learn how to use regularities in the environment, humans use a form of error-driven task learning, where the output (action) that is produced is monitored and slowly adapted towards the desired output.

Following the human visual system, we employ a combination of model-based learning and error-driven task learning. Model-based learning is well suited for extracting cloud patterns in the satellite images, while error-driven task learning is necessary for training the network to forecast a correct cyclone track direction on the basis of the shape and orientation of the detected cloud patterns. One way of combining the two learning algorithms would be to let the first layers in the network perform model-based learning, while subsequent layers would use error-driven task learning to tune the weights towards achieving the task at hand. However, if weights were changed independently in the first few layers, these weights could develop to work against the required input-output mapping. A more efficient way to combine the two learning algorithms is to let both take place in parallel at each layer, and to simply combine the outcome of the two algorithms for each learning cycle at each layer. There are both practical (faster learning) and theoretical gains (biological plausibility) in using this combination of learning algorithms (cf. O’Reilly, 1998). 


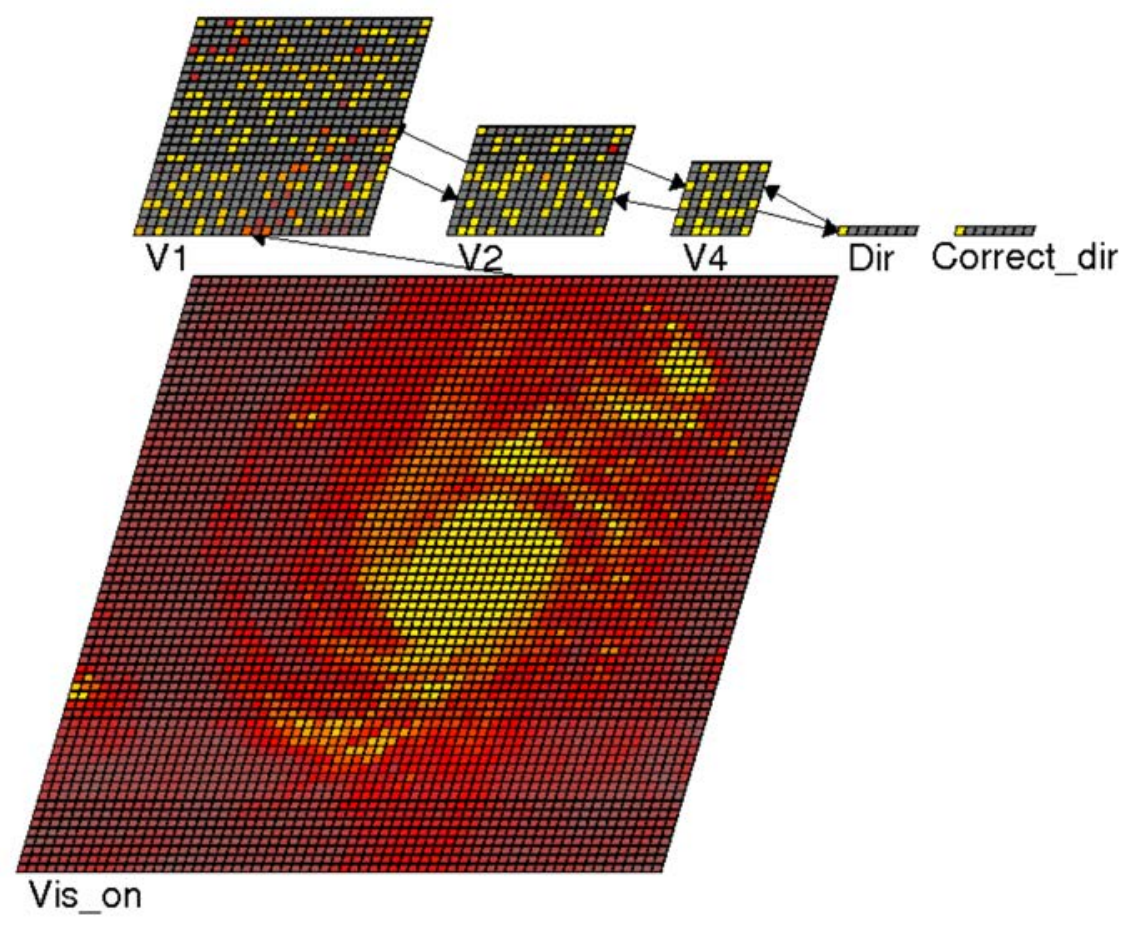

Fig. 2. The five-layer network used in this study, with bi-directional connections going between groups of sending and receiving nodes. Hence, the network is constructed to detect constituent features in limited parts of the satellite image, and then integrate these features into an overall could pattern. Note that the layer called 'Correct_dir' did not partake in computation; it was used to display the correct answer, which enabled us to visually compare predicted versus correct direction of cyclone movement. In addition, the difference between output and correct direction was calculated as the summed squared error, and this measure was used for performance analysis and for training the network.

\subsection{Biological transformational steps}

At the first transformational step in the human visual system (Fig. 3), the image captured by the retina is routed via the Lateral Geniculate Nucleus (LGN) to primary visual cortex (brain area $\mathrm{V} 1$ ). More exactly, the role of LGN is to merge input from the two eyes and to mediate this combined information to primary visual cortex V1. 


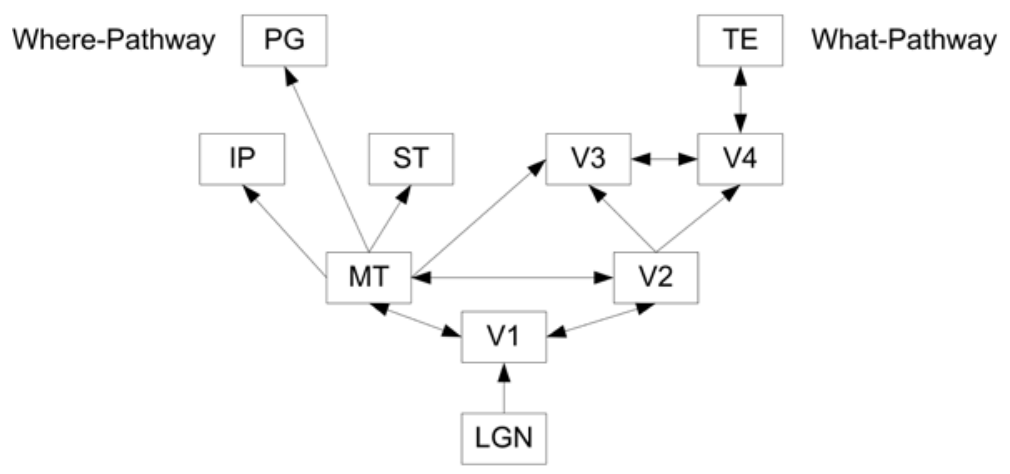

Fig. 3. Architecture of the human visual system. The what- and where-pathways depicted in the figure are physically separated in the brain. The where-pathway runs in the dorsal (upper) part of the brain, and is specialized for spatial processing of size, location and orientation of objects. The what-pathway is located in the ventral (lower) part of the brain, and implements color and shape detection (figure adapted from Felleman and Van Essen, 1991).

Brain area V1 contains specialized neurons that are sensitive to specific features, such as variously oriented line segments. ${ }^{1} \mathrm{~V} 1$ neurons are dedicated to process input from a delimited part of the retinal image, which in turn corresponds to a specific region in external space. This region comprises the neuron's receptive field (RF).

Feature detector neurons with the same RF cooperate in detecting various features at the same part of space. For example, neurons that cover the same part of space may be sensitive to line segments with various orientation; these neurons are organized into one hyper column. Neighboring hyper columns of feature detectors cover neighboring parts of space. Together, the matrix of hyper columns can process the entire input image. Logically, the simultaneous activation of a specific combination of neurons within these groups would reveal the presence of various features at specific parts of the image.

However, image interpretation is not accomplished in one step in the brain, as it would be extremely difficult to reliably recognize a complex pattern of features, when feature detection itself is underdetermined and has to be adjusted to variations in appearance (size, location, and orientation) of these features. There are simply too many degrees of freedom and these cannot be handled all at once. Instead, the brain implements a gradual transformation, cutting down the space of possible interpretations of the input image step by step. Hence, neighboring simple features represented in V1 are merged into slightly more complex (aggregate) features at the next step of processing that is, in brain area V2. More specifically, detectors for these aggregate features react to the combined activation of simple feature detectors in V1. Also V2 neurons receive input from a limited part of the retinal image, but these RFs now cover a slightly larger part of space.

Through further stepwise transformations (in areas V3, V4 and TE), features are aggregated into more and more complex object properties. For each transformational step, the aggregated

${ }^{1}$ Visual features constitute the basic building block of human vision, and reflect simple visual properties that are present in limited parts of the image. For example, recognition of the letter 'A' builds on detection of the constituent line segments ' $\%$ ' ' ' and ' - '. These features are located at different parts of the input (the left, right, and middle part of the input). It is the combined presence of these features and their relative location that signals the presence of the letter 'A'. 
features will originate from larger and larger parts of the image. At each transformational step, feature detection needs only to allow for variations in location within a limited part of space (i.e. within the feature detector's RF). Likewise, processing is simplified as variations in size and orientation apply to simpler constituent features (as opposed to complex overall patterns). In addition, variations in size are limited by the boundaries of the RF. Hence, step-wise processing makes handling of the many degrees of freedom inherent to image processing more tractable.

\subsection{Architecture of the artificial neural network}

Robust pattern recognition can thus be achieved through stepwise processing in a bi-directionally connected multi-layer network. At the first level, shape recognition is based on detection and integration of characteristic constituent features, such as small cloud segments. The constituent features are smaller in size than the aggregate shape (the whole cloud pattern), and therefore can be detected by looking at limited parts of the image.

The stepwise integration of features that we have implemented in our artificial neural network resembles the part of the human visual system which is responsible for pattern detection and shape recognition, what is commonly called the what-pathway (Goodale and Milner, 1992; Kosslyn, 1994; O’Reilly, 1998; Creem and Proffitt, 2001).

By processing the image through a matrix of feature detector units, constituent features in different parts of the image can be extracted in parallel (O’Reilly and Munakata, 2000). For each feature that is detected, the network needs to abstract away from minor variations in position and size, but only within a small part of the image. Due to the task requirements, rotational variations are encoded as separate features at each stage of processing.

At subsequent steps of processing, the simpler constituent features are integrated into larger patterns that is, into more complex features. Recognition of these secondary features is based on the combined activation of feature detectors at the previous transformational stage, and is thus not strictly dependent on which part of the image these activations has arisen from. For each additional transformational step, the more independency is achieved and the more complex patterns can be recognized.

At the final stages of processing, the constituent features can be integrated into an overall shape which is rotated in a specific way. This information is then mapped onto a directional indicator showing the predicted movement of the cyclone.

\subsubsection{Enhanced generalization ability}

Through consecutive transformational steps, detection of shape and orientation becomes increasingly independent of the exact position and size of the features, and is instead based on how the constituent features are oriented, located, and sized relative to each other.

Image processing is based on constituent features which may be present in many satellite images. Novel satellite images that have not been presented to the network previously, could still elicit a correct response based on the novel combination of previously experienced and well learnt features. Hence, step-wise image processing enhances the network's ability to generalize to novel images.

\section{Implementation of the network}

The network presented in this paper was implemented and trained using the artificial neural network simulation tool Leabra++, which is specialized for modeling biologically-based (brainlike) artificial neural networks (O’Reilly and Munakata, 2000).

The network consisted of an input layer, three consecutive hidden layers, and an output layer (Fig. 2). Layers were bi-directionally connected, which meant that if a layer was sending input to another layer, it also received feedback from that layer. Connections were symmetrical, so that is 
unit $x$ was sending input to unit $y$, then $\mathrm{x}$ also received feedback from $y$. This symmetry was preserved during training.

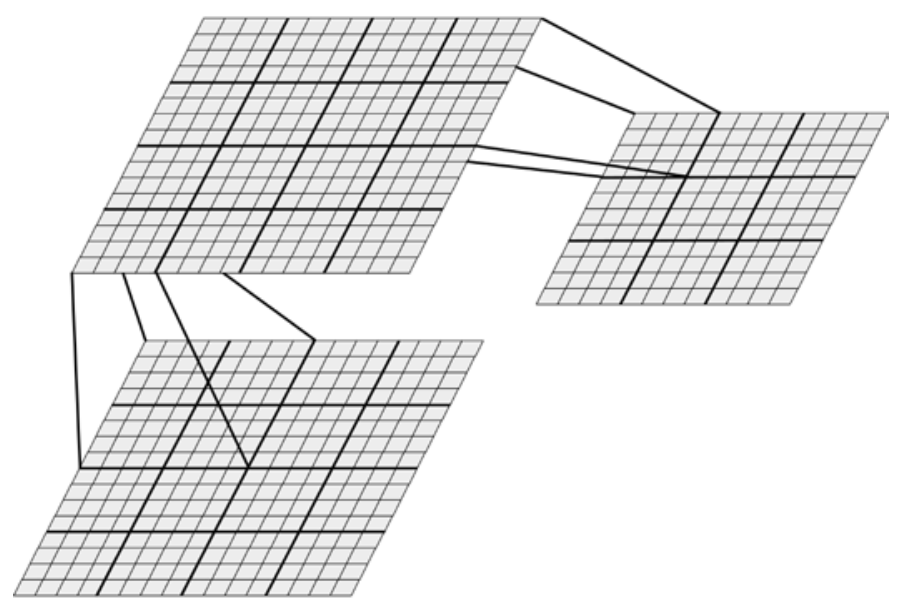

Fig. 4. Selective communication between corresponding groups of nodes across layers, resembling the limited receptive fields in the human visual system. For each transformational step, increasingly complex features can be extracted, and these features originate from increasingly larger parts of space.

The first receiving layer V1 was designed to encode simple visual features in the input image, such as small pieces of cloud patterns. To be able to extract features from part of the input, nodes in this layer were grouped and connected to the input in a way so that each group would receive input only from a limited part of the image. Grouping of nodes and selective communication between groups of nodes across layers simulated the concept of limited receptive fields (RF) that can be found in the human visual system (Fig. 4). Likewise, each group in the sending layer received feedback signals from a small number of neighboring groups in the receiving layer. Neighboring receptive fields slightly overlapped, in order to maintain continuity of processing across various parts of the image.

In the process of training we found that the RF size is critical for achieving fast and stable learning (i.e. independent of initial weights). V1 therefore consisted of 25 groups of 8 x 8 units each. Each of these groups received input from approximately $1 / 25^{\text {th }}$ of the input image (overlaps disregarded).

Using this network architecture, local information from different parts of the input image could be processed in parallel. For each transformational step in the network, information was integrated, taking into account larger and larger parts of the input image. Hence, layer V2 consisted of 16 groups of 8 x 8 units, where each group received input from 4 groups in layer V1. Layer V3 contained 4 groups of $8 \times 8$ units, where each group received input from 9 groups in layer V2. Finally, layer V4 comprised of 16 x $16=256$ units, where each unit received input from all units in V3. All feedback connections were symmetrical on a unit-to-unit level.

At the final transformational step (V4 $\rightarrow$ Dir), the shape and orientation of the overall cloud pattern was transformed into a directional prediction, indicating in which direction the cyclone was most likely to move. Output from the network was represented by an $8 \times 1$ vector, where each bit (i.e. activation of one individual node) signified a possible rotational direction ranging from $0^{\circ}$ to $315^{\circ}$ in $45^{\circ}$ steps. The low resolution of output reflected the relatively low resolution of the input images (66 x 66). 


\subsection{Training data}

Training data was prepared by downsizing the images to $66 \times 66$ pixels and then converting the satellite images into grayscale and linearly transforming this image from a format where each pixel contained a value between 0 and 255 into a representation where pixel values were limited to the interval $0-1$. This transformation would thus map an original pixel value of 255 to a new pixel value of 1 . Similarly, an original pixel value of 127 would be transformed into a new pixel value of $127 / 255$, and so on. Note that this transformation preserved the information contained in the original image.

The resulting image was then artificially rotated clockwise in $45^{\circ}$ steps, which resulted in 8 rotated images.

In order to create variation in the cyclone's size relative to the input image frame, each of the rotated images was zoomed in three ways (using a zoom factor of $0.8,1$, and 1.2 respectively).

We were forced to use relatively low-resolution images (Fig. 5) due to computer power limitations. We used commercially available standard PCs for our network simulations. We reasoned that if the network would learn to forecast cyclone tracks based on these low-resolution images, this would be an excellent proof of concept, demonstrating the usefulness and robustness of ANN-techniques for satellite image interpretation.

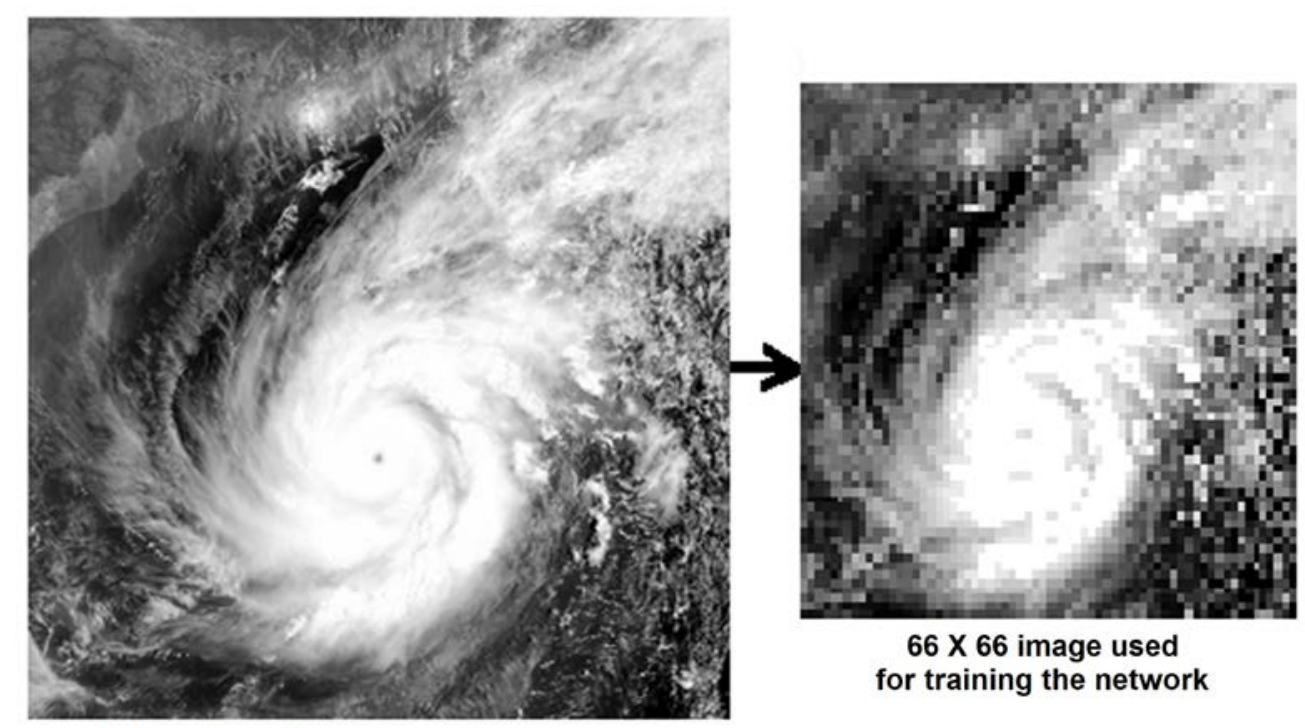

NOAA-AVHRR image of cyclone Mala

Fig. 5. Example of original image and corresponding downsized image that was used to generate input patterns to the network. The downsized image was artificially rotated clockwise in $45^{\circ}$ steps. Each of the rotated images was zoomed and shifted in position, in order to create variation in the cyclone's orientation, size, and location in the image.

Finally, each image was shifted in position zero, one, or two steps (where each step equaled three pixels) using all combinations of directions (north, south, east, west, northeast, et cetera) in order to provide the network with variation in the position of the cyclone relative to the image frame. As cyclone position could differ by as much as 12 pixels, that is, almost one fifth of the total image size, important information concerning the cyclone's surroundings could disappear off the edge for some images. To avoid moving the most informative part of the cyclone's surroundings outside the image frame, certain combinations of image rotation and two-step 
position shifts were automatically discarded. All in all, these transformations resulted in about 1200 images.

From the total set of images, a random set of approximately $5 \%$ of the images was set aside for later testing of network performance. The remaining $95 \%$ of the images were used for training of the network.

Correct movement direction for the images was determined by visual estimation of the relative position of cumulonimbus clouds in the surrounding the cyclone. This procedure was based on the rule of thumb that the cyclone is most likely to move in a direction where there are cumulonimbus clouds in the outer circulation bands around the cyclone (cf. Fig. 1). The network's task was to detect the same pattern of cyclone with surrounding cumulonimbus clouds that the human eye did, and use this information to predict the cyclone's future movement direction. In this way, the artificial neural network was required to play a role similar to a human expert when extracting information from satellite images.

\subsection{Information processing in the network}

For each input-output pair in the training set, the network went through two activation phases. During the minus phase the network was presented with the input and was allowed to settle in an activation state consistent with this input and with the weights in the network. During settling, weakly activated nodes were suppressed, allowing at most $k$ nodes to be active within a group of nodes in the same layer. This mechanism, referred to as $k$-winners-take-all (kWTA) allowed only a predetermined number of nodes to be active within each layer.

In the second plus phase activation in the network was reset to zero and the network was presented with both the input and the correct output. In this second phase, the network settled into an activation state that reflected the correct activation of each node, given the correct output. By comparing the activations between the minus and the plus phases, an error was computed for each node.

The incoming weights for each node were adjusted in a direction that decreased this error. In addition, a weight change was also computed based on co-activation of pair of nodes: Nodes that were activated together during the plus phase were assigned a small positive weight change to enforce this tendency for co-activation. Likewise, a negative weight change was assigned to connections leading from an inactive to an active node, and vive versa, to decrease the dissonance between these nodes. The final weight update for each connection reflected the sum of the error-driven and correlation-based weight change (see Equation 1).

After each epoch that is, after the complete training was presented to the network (each time in permuted order), an average error was calculated. The average error was recorded and plotted for each epoch (i.e. after each presentation of the complete training set).

Training of the network can be described in mathematical terms as follows. Weights in the network were limited to the interval [0 1]. Also the activation of nodes was limited to the interval [0 1]. The activation function (describing how strongly a node should react to input) was given by the following biologically-based, sigmoid-like function (O’Reilly \& Munakata, 2000):

$$
\begin{gathered}
y_{j}=\frac{\gamma\left[V_{m}-\Theta\right]_{+}}{\gamma\left[V_{m}-\Theta\right]_{+}+1}, \quad[z]_{+}=\left\{\begin{array}{l}
z \text { if } z \geq 0 \\
0 \text { if } z<0
\end{array}\right. \\
y_{j}=\text { activation of receiving node } j \\
\gamma=\text { gain } \\
V_{m}=\text { membrane potential } \\
\Theta=\text { firing threshold }
\end{gathered}
$$


Learning in the network was based on a combination of Conditional Principal Component Analysis (CPCA), which is a form of unsupervised model-based learning algorithm (Hebbian learning) and Contrastive Hebbian learning (CHL), which is a biologically-based error-driven algorithm, an alternative to backpropagation of error (O’Reilly, 1998; O’Reilly \& Munakata, 2000):

$$
\begin{aligned}
\text { CPCA: } \Delta w_{i j}=\varepsilon y_{j}\left(x_{i}-w_{i j}\right)=\Delta_{h e b b} \\
x_{i}=\text { activation of sending node } i \\
y_{j}=\text { activation of receiving node } j \\
w_{i j}=\text { weight from node } i \text { to node } j
\end{aligned}
$$

$$
\begin{aligned}
\text { CHL: } \Delta w_{i j}= & \varepsilon\left(x_{i}^{+} y_{j}^{+}-x_{i}^{-} y_{j}^{-}\right)=\Delta_{e r r} \\
& x_{i}=\text { activation of sending node } i \\
& y_{j}=\text { activation of receiving node } j \\
& x^{+}, y^{+}=\text {activations when both input and correct output is presented to the network } \\
& x^{-}, y^{-}=\text {activations when only input is presented to the network }
\end{aligned}
$$

Combined learning: $\Delta w_{i j}=\varepsilon\left[\mathrm{C}_{\text {hebb }} \Delta_{\text {hebb }}+\left(1-\mathrm{C}_{\text {hebb }}\right) \Delta_{e r r}\right]$

$\varepsilon=$ learning rate

$\mathrm{C}_{\text {hebb }}=$ proportion of Hebbian learning (set to 0.01 in the present study)

\section{Results}

Training was run in Epochs. Each epoch consisted of one round of presentation of the complete training set of approximately 1125 images (around $95 \%$ of all available images; a random $5 \%$ was saved for testing). During the training of each epoch, the images were permuted, to avoid sequence learning effects.

Error in network performance was defined as the difference between correct output and the output produced by the network, calculated for each output unit, and squared and summed into an overall error measure. The output layer in the network consisted of 8 nodes, where each node represented a possible movement direction, ranging from $0^{\circ}$ (north) to $315^{\circ}$ (northwest) in $45^{\circ}$ steps. Performance error was measured in each of these eight nodes, as the network was required to activate exactly one of these nodes, but not the others. The individual errors were squared (to eliminate differences in sign) and summed, producing a summed squared error (SSE) for each output produced by the network. Performance error for each epoch was measured as the SSE summed over all input-output pairs in the training set. We conducted multiple trainings to make sure that network behavior was stable, that is, independent of the initial random weights. In general for all training runs, training error (SSE) decreased rapidly and leveled out at around 20 after 10-15 epochs of training (Fig. 6). This meant an average error of 0.02, divided among 8 nodes, for each output produced by the network.

These results were attained for images where the position of the cyclone center was varied by $6 \%$ of the total image size. This means that when using the network for cyclone track prediction, the cyclone needs not be precisely positioned in the center of the image in order for the network to produce valid track predictions. To get a feeling for how well the network handled variations in the position of cyclone center, we experimented with training the network using larger variations in the position of cyclone center. A typical training curve for such a training set is depicted with dashed line in Fig. 6. 


\section{Training error}

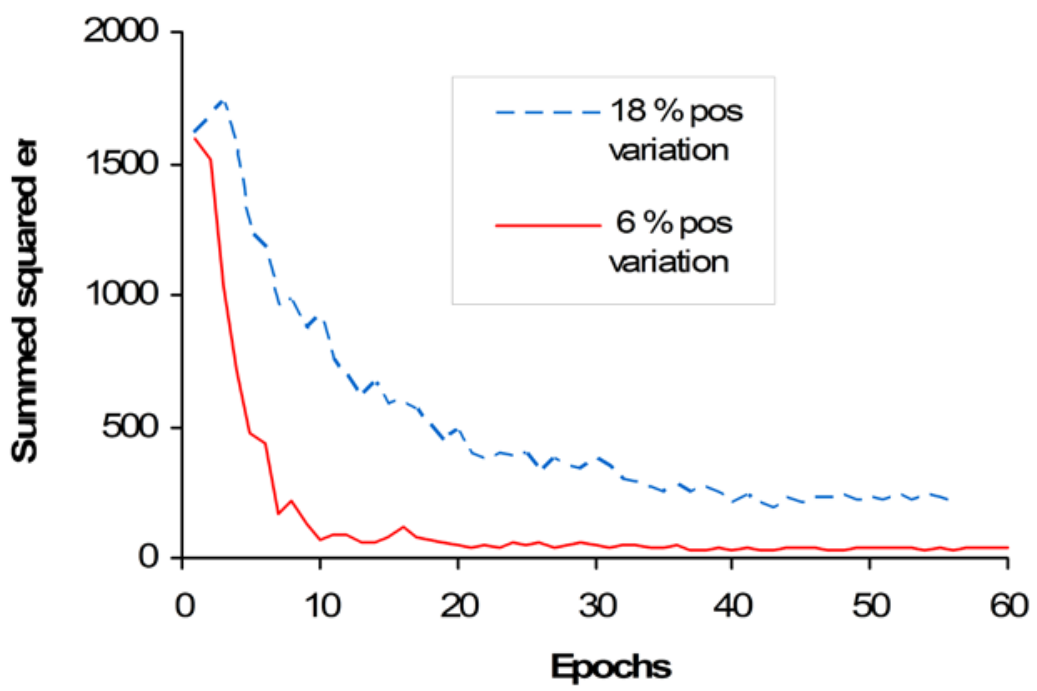

Fig. 6. Sample training curves. Typically, the network reached a training error of 30-40 after 10-15 epochs of training, when cyclone position was varied by $6 \%$ of the image size. The dashed line shows a sample training curve for input images where the cyclone center varied in position by up to $18 \%$ of the image size.

\subsection{Network performance for novel images}

About $5 \%$ of the images were excluded from the training set and were used as test images. These test images were presented to the network for the first time during testing. During testing of the networks, we recorded which images were and were not interpreted correctly. We obtained about 99 \% correct cyclone movement direction prediction for these novel cyclone images when
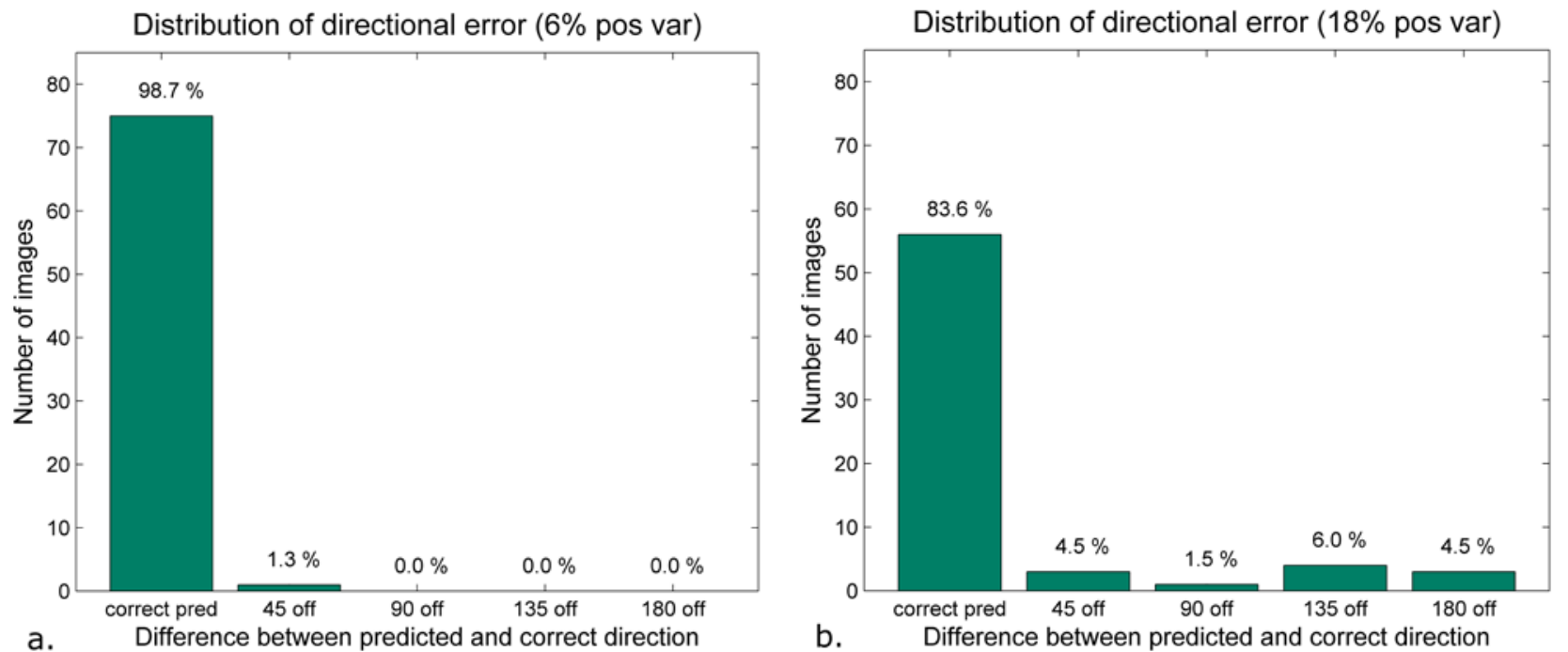

Fig. 7. Sample distribution of errors for the test images. a. Directional errors when cyclone center varied by $6 \%$ of the total image size. The leftmost bar indicates correct cyclone track predictions for novel images $(98.7 \%)$. b. Distribution of errors when cyclone position varied by $18 \%$. A stable pattern across multiple runs was the relatively few orthogonally incorrect predictions ( $90^{\circ}$ off).

cyclone center varied by $6 \%$ of the image size (Fig. 7 a). When the position of the cyclone center varied more within the image frame (18\% of the image size), correct track predictions dropped to $84 \%$ for novel images (Fig. 7 b). 
For the image set that contained images with up to $18 \%$ positional variance of the cyclone center relative to the image frame, several training and testing sessions were conducted using different starting weights, and we detected a reoccurring pattern. In about $5 \%$ of the cases that is, for 3-4 of the 70-75 images, the network mistakenly predicted that the cyclone would move opposite to the correct direction. In other words, the prediction was $180^{\circ}$ off (Fig. 7 b). For a couple of images, prediction was $45^{\circ}$ off. Finally, for 1-2 images predicted direction was perpendicular to the correct direction. Hence, mistaken predictions tended to be either close to the correct direction ( $45^{\circ}$ off), or in the opposite direction (and directions close to this). In contrast, incorrect predictions that lay perpendicular to the correct cyclone track were comparatively rare.

\section{Analysis of network performance}

The pattern of errors produced by the network (Fig. 7) indicated that predictions were based on shape information, namely the elongated shape formed by the cyclone together with the cumulonimbus clouds on one side of the cyclone. The images fed into the network were relatively low-resolution ( $66 \times 66)$, and it seems that for some of these images, the network mistakenly recognized the elongated shape as being upside-down, or mirrored left-to-right, resulting in predictions that were approximately $180^{\circ}$ off. In contrast, the network made relatively few errors where predictions were $90^{\circ}$ off. 

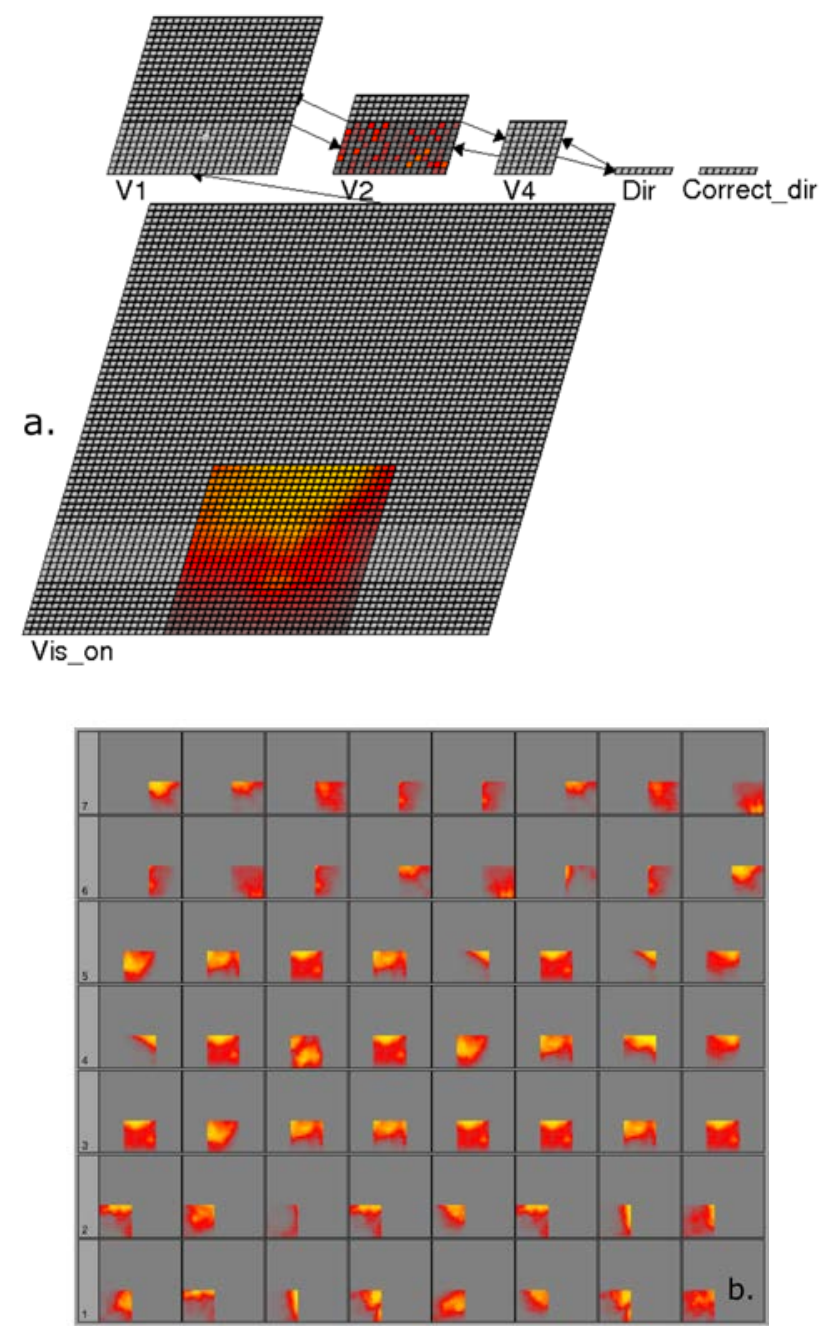

Fig. 8. a. Receiving weights developed during training belonging to a particular node in V1. This node receives input from part of Vis_on (the RF for this node), and also feedback signals from V2. Note that weight pattern from Vis_on resembles a cloud segment—-this is the feature that this particular receiving node specializes for. b. Sample incoming weights for neighboring receiving nodes in V1. Square $(\mathrm{x}, \mathrm{y})$ shows the weights from part of Vis_on into node $(x, y)$ within a receiving group in V1. Note that some nodes (e.g., node $(4,0)$ have evenly distributed random weights after training, meaning they have not specialized for any feature. Even if these nodes do not participate in final computation, they provide useful elbow-room during competitive learning.

The internal workings of artificial neural networks are often considered to be impenetrable for the human analyst. An interesting question is therefore how the network performs cloud pattern recognition. For this, we need to understand which low-level features are extracted by the network when a satellite image is presented. What sort of low-level features the network has learnt to detect is in turn reflected by the weight structure that has developed during training between the first input layer (Vis_on) and the next receiving layer (V1).

The receiving (incoming) weights into individual receiving units in V1 can be plotted to see which pixels in the input image that this particular receiving unit has learnt to focus on. An example is shown in a, where the receiving weights have been visualized for one unit in V1. As 
can be seen in the figure, these weights indicate that the receiving unit has specialized in detecting a shape that resembles a cloud segment.

One way of visualizing the receiving weights for several units is to stack the weight matrices for neighboring receiving units. Hence, for example, the weight matrix for unit $(0,0)$ in V1--- the leftmost bottom unit---would be placed in position $(0,0)$ in the stacked weight matrices plot. Looking at the stacked weight matrices plot, it becomes evident that various receiving units in V1 have specialized for detecting different types of cloud segments. Depending on which of these features are present in the satellite image, various receiving units in V1 will be activated simultaneously, indicating the combination of features. Based on this activation pattern, subsequent layers (e.g. V2) will be able to recognize the original cloud shape.

\section{Discussion}

The network was able to learn the task quickly (in 15-20 epochs), which indicates that the network architecture (size and structure of layers and connection structure) was correctly set.

Because we had limited computational power available, we were forced to use relatively low-resolution input images (cf. Fig. 5). At this level of resolution, there is a risk that elongated shapes are perceived as equally thick at both ends, making it impossible to distinguish the cyclone itself from neighboring cumulonimbus clouds. It thus becomes difficult to determine which end of the elongated shape is occupied by the cyclone, and which part constitutes the surrounding cumulonimbus clouds.

In spite of the low-resolution images (66 x 66 pixels) that were used, it was possible to train the network and achieve correct cyclone track predictions in $99 \%$ of the cases, when small variations in the cyclone position within the image were allowed. According to our analysis, the network was able to learn to interpret the shape of cloud patterns in the cyclone, and map the orientation of this overall shape to a movement direction. Most importantly, the network could achieve this irrespective of variations in position and apparent size of the cyclone.

When larger variations in cyclone position were allowed, for about $10 \%$ of the images, the network mistakenly interpreted the image as being upside-down or mirrored, making predictions that were $180^{\circ}$ off. This type of error entails that the network successfully recognized the elongated shape that was formed by the cyclone and the surrounding clouds, but that the orientation of this overall shape was mistakenly recognized as being upside-down or left-right mirrored. These mistakes are most likely caused by the low-resolution images that were used as input.

The present study is a proof of concept and is meant to demonstrate the applicability of neural network techniques for cyclone track prediction. This work constitutes a first step towards fully automated interpretation of satellite images for the purpose of cyclone track and intensity prediction. The technique that we propose remains to be tested on higher resolution images of a wide variety of cyclones. However, we have to note that processing of high-resolution images in an artificial neural network of this size most probably requires parallel computing resources, such as a cluster of PCs using distributed memory.

\section{Future work}

The results that we have obtained are promising. To the extent that cyclone movement direction is reliably indicated by satellite data in the visible range, neural network techniques seem to be effective in extracting this information.

The kind of deep recurrent (bi-directionally connected) network that we have used could easily be redesigned to take into account also other non-visual factors, such as previous cyclone track and current movement direction of the cyclone, as well as temperature and air pressure data. Inclusion of supplementary input to the network would impose additional constraints which 
would entail improved guidance of activation settling in the network, and hence improved predictions.

We see several avenues for future work. First of all, it would be interesting to develop a scaled-up version of the network, and compare its performance with the present network to see if higher resolution images contain additional information that is useful for recognizing cloud patterns and determining movement direction. Although our present training results indicate that low-resolution images may be sufficient in some cases, we expect that many cloud patterns, especially at the early stages of cyclone development, may be too small or too finely structured to be detectable in a low-resolution satellite image.

We would also like to include additional information as input to the network, and train the network to produce practically useful prediction of future cyclone track. Network performance could then be evaluated on the basis of a series of images of past cyclones, by comparing actual development of the cyclone track with predictions produced by the network. After training and validation of the network using historical data, the network will have encoded statistical knowledge of the relationship between cloud patterns surrounding the cyclone and likely cyclone track. This statistical knowledge could be set to work when predicting the track of new cyclones.

\section{References}

Barsi, A., and Heipke, C., 2003a. Artificial neural networks for the detection of road junctions in aerial images. International Archives of Photogrammetry, Remote Sensing and Spatial Information Sciences 34 (Part 3/W8), 113-118.

Barsi, A., and Heipke, C., 2003b. Detecting road junctions by artificial neural networks

Brad, R., and Letia, I.A., 2002a, Extracting cloud motion from satellite image sequences, Seventh International conference on control, Automation, Robotics and Vision (ICARCV'2002), Singapore.

Brad, R., and Letia, I.A., 2002b, Cloud motion detection from infrared satellite images, The International Society for Optical Engineering (SPIE), Second International Conference on Image and Graphics, 4875, 408-412.

Bureau of Meteorology Research Centre, 2006, Global guide to Tropical Cyclone forecasting, Australia. http://www.bom.gov.au/bmrc/pubs/tcguide/globa_guide_intro.htm Visited 200701-12.

Cerveny, R. S. and Newman, L. E., 2000, Climatological relationships between tropical cyclones and rainfall. Monthly Weather Review, 128, 9, 3329-3336.

Creem, S. H., and Proffitt, D. R., 2001, Defining the cortical visual systems: "What", "Where”, and "How", Acta Psychologica, 107, 1-3, 43-68.

Dvorak, V.F., 1975, Tropical Cyclone Intensity Analysis and Forecasting from Satellite Imagery, Monthly Weather Review, 103, 5, 420-430.

Felleman, D. J., and Van Essen, D. C., 1991, Distributed hierarchical processing in the primate cerebral cortex. Cerebral Cortex, 1, 1-47.

Fett, R.W. and Brand, S., 1975, Tropical cyclone movement forecasts based on observations from satellites, Journal of Applied Meteorology, 14, 4, 452-465.

Goodale, M. A., and Milner, A. D., 1992, Separate visual pathways for perception and action. Trends in Neuroscience, 15, 1, 20-25.

Hong, Y., Hsu, K.-L., Sorooshian, S., Gao, X., 2004, Precipitation Estimation from Remotely Sensed Imagery using an Artificial Neural Network Cloud Classification System, Journal of Applied Meteorology, 43.

Jang, J.-D., Viau, A.A., Anctil, F., and Bartholomé, E., 2006, Neural network application for cloud detection in SPOT VEGETATION images, International Journal of Remote Sensing, 27, 4, 7 19-736 
Kishtawal, C. M., Patadia F., Singh R., Basu S., Narayanan M. S., and Joshi P. C., 2005, Automatic estimation of tropical cyclone intensity using multi-channel TMI data: A genetic algorithm approach, Geophysical Research Letters, vol 32.

Knaff, J. A., Zehr, R. M., Goldberg, M. D., 2000, An example of temperature structure differences in two cyclone systems derived from the advanced microwave sounder unit. Weather and Forecasting: 15, 4, 476-483.

Kossin J., 2003, A user's guide to the UW-CIMSS Tropical Cyclone Intensity Estimation (TIE) Model. Cooperative Institute for Meteorological Satellite Studies (CIMSS), University of Wisconsin-Madison.

Kosslyn, S. M., 1994, Image and Brain: The resolution of the imagery debate. Cambridge MA, MIT Press.

Lau, N. C. and Crane, M. W., 1997, Comparing satellite and surface observations of cloud patterns in synoptic-scale circulation systems. Monthly Weather Review, American Meteorological Society, 125, 12, 3172-3189.

Lajoie, F. A., 1976, On the direction of movement of tropical cyclones, Australian Meteorological Magazine, 24, 95-104.

Marshall, J. F. L., Leslie, L. M., Abbey Jr. R. F., and Qi, L., 2002, Tropical cyclone track and intensity prediction: The generation and assimilation of high-density, satellite-derived data. Meteorology and Atmospheric Physics, 80, 1-4, 43-57.

McBride, J.L., Holland, G.J., 1987, Tropical-Cyclone Forecasting: A Worldwide Summery of Techniques and Verification Statistics, Bulletin of the American Meteorological Society, 68, 10, 1230-1238.

Naval Research Laboratory, 1999, Tropical Cyclone Forecasters Reference Guide, http://www.nrlmry.navy.mil/ chu/ Visited 2007-01-12.

O’Reilly, R. C., \& Munakata, Y., 2000, Computational explorations in cognitive neuroscience: Understanding the mind by simulating the brain. Cambridge, MA: MIT Press.

O’Reilly, R. C., 1998, Six principles for biologically-based computational models of cortical processing. Trends in Cognitive Sciences, 2, 11, 455-462.

Rivolta, G., Marzano, F.S., Coppola, E., and Verdecchia, M., 2006, Artificial neural-network technique for precipitation nowcasting from satellite imagery, Advances in Geosciences, 7, 97-103.

Ungerleider, L. G., and Mishkin, M., 1982, Two cortical visual systems. In Ingle, D. J., Goodale, M. A., and Mansfield, R. J. W. (eds): The analysis of visual behavior. Cambridge, MA: MIT Press.

Velden, C.S., Olander, T.L., and Zehr, R.M., 1998, Development of an Objective Scheme to Estimate Tropical Cyclone Intensity from Digital Geostationary Satellite Infrared Imagery, Weather and Forecasting, 13, 1, 172-186.

Wells, F. H., 1987, Tropical cyclone intensity estimates using satellite data: the early years. Proceedings of 26th Conference on Hurricanes and Tropical Meteorology. 